\title{
A Case of Popliteal Artery Entrapment Syndrome with Chronic Total Occlusion
}

\author{
Yasunori Iida, MD, Ph.D, ${ }^{1}$ Yukio Obitsu, MD, Ph.D, ${ }^{1}$ Naozumi Saiki, MD, ${ }^{1}$ Miki Izumi, MD, Ph.D, ${ }^{2}$ \\ Hiroyoshi Komai, MD, Ph.D, ${ }^{1}$ and Hiroshi Shigematsu, MD, Ph.D ${ }^{1}$
}

\begin{abstract}
Popliteal artery entrapment syndrome (PAES) is rare congenital anomaly that occurs due to compression of the popliteal artery by adjacent musculotendinous structures. We report a 54-year-old woman with PAES of total popliteal arterial occlusion was successfully treated by release of the muscle bundle and reconstruction of the popliteal artery. Pathologic examination revealed that the extracted portion of the popliteal artery had chronic total occlusion with fibrosis and destruction of internal elastic membrane. We should deliberate whether we reconstruct the popliteal artery in addition to release of the aberrant muscle bundle due to the preoperative examination to prevent the reoperation.
\end{abstract}

Key words: popliteal artery entrapment syndrome, chronic total occlusion, CT angiography

\section{INTRODUCTION}

$\mathrm{P}_{\mathrm{c}}^{\mathrm{s}}$ opliteal artery entrapment syndrome (PAES) is a rare congenital anomaly that occurs due to compression of the popliteal artery by adjacent musculotendinous structures, which was first described by Stuart.' ${ }^{1)}$ The diagnosis is usually made by computed tomography (CT) or magnetic resonance imaging (MRI), and, in particular, computed tomographic angiography (CTA) can express the precise anatomical relations of muscle, bone and artery as well as the degree of stenosis or occlusion of the popliteal artery. CTA is helpful when we bypass the popliteal artery in addition to releasing the aberrant muscle bundle.

${ }^{1}$ Department of Vascular Surgery, and ${ }^{2}$ Department of Medical Education, Tokyo Medical University, Tokyo, Japan

Received: April 2, 2010 Accepted: June 28, 2010 Address for reprint requests to Yasunori Iida, MD, Ph.D: Department of Vascular Surgery, Tokyo Medical University, 6-7-1 Nishishinjuku, Shinjuku-ku, Tokyo 160-0023, Japan Tel: +81-3-3342-6111 (Ext 5077), Fax: +81-3-3342-6193

E-mail: y.iida@vanilla.ocn.ne.jp

\section{Case Report}

A 54-year-old woman presented complaining of severe pain in the right calf when mountaineering and she gradually felt deterioration of the right lower limb fatigue on exertion. Systemic examinations, which included blood pressure and other laboratory investigations such as atherosclerotic risk factors were unremarkable. The preoperative right ankle brachial pressure index (ABPI) dropped from 0.9 to "immeasurable" on ankle flexion. CTA demonstrated segmental occlusion of the right popliteal artery and genicular collateral developments (Fig. 1). We also confirmed right popliteal artery extrinsically compressed by gastrocnemius muscle in the horizontal image of CT. Diagnosis of PAES was made and surgical release of the aberrant gastrocnemius muscle bundle and popliteal arterial reconstruction using the great saphenous vein were performed because we recognized complete occlusion of the right popliteal artery on CTA. Pathologic examination revealed that the extracted portion of the popliteal artery had chronic total occlusion with fibrosis and destruction of the internal elastic membrane (Fig. 2, asterisk). The postoperative ABPI rose from "immeasurable" to 1.28 even on ankle flexion. There were no abnormal findings in the postoperative CTA, the patient was discharged and she is now able to 


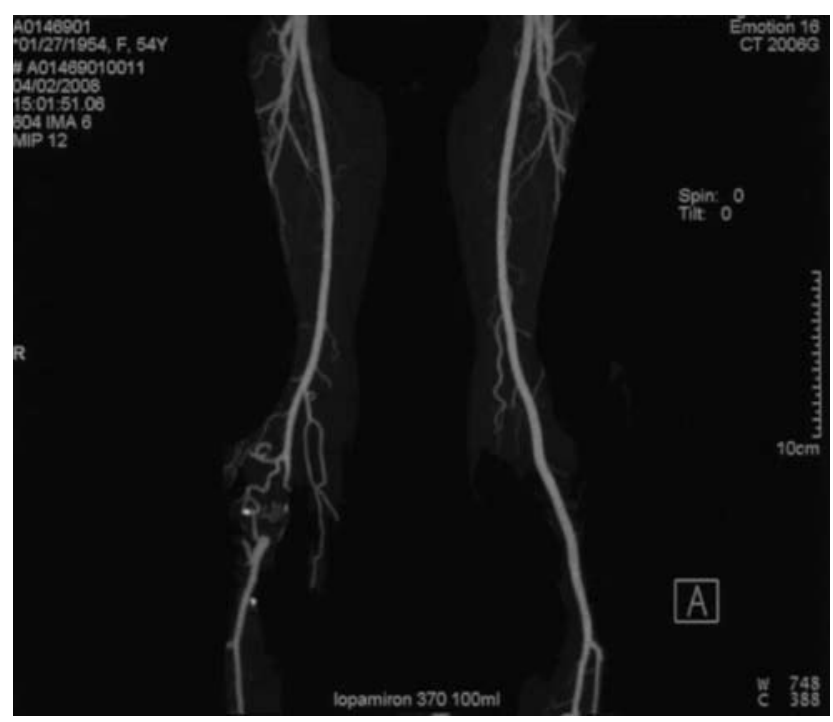

Fig. 1 Preoperative CTA demonstrates segmental occlusion of the right popliteal artery and genicular collateral developments.

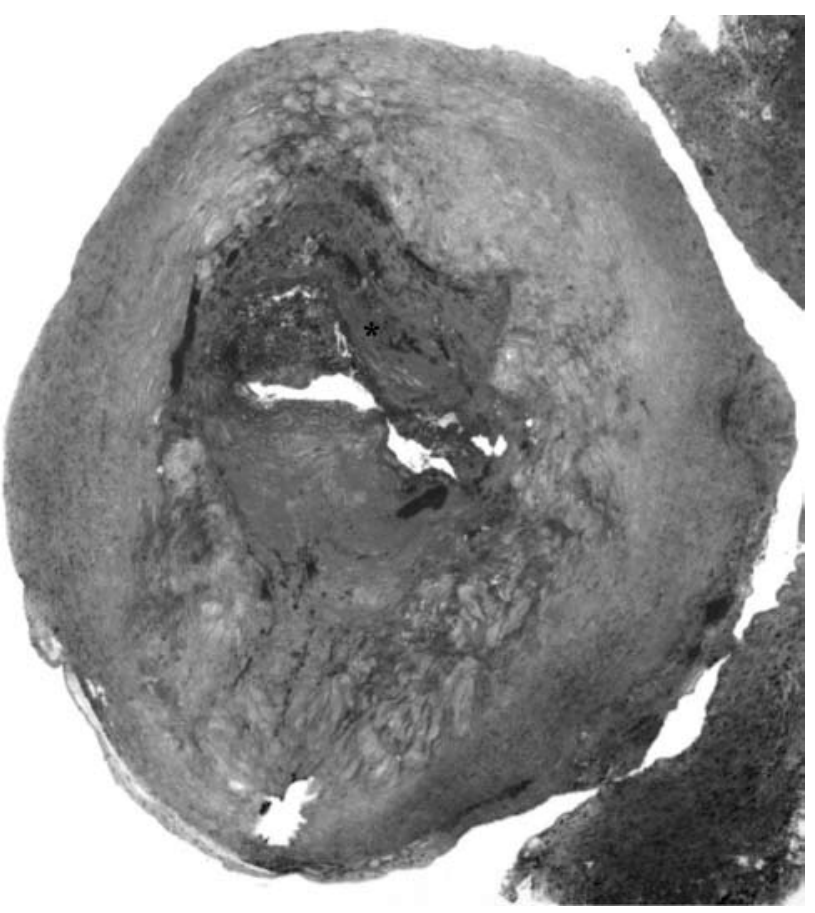

Fig. 2 The extracted portion of the popliteal artery has chronic total occlusion with fibrosis and destruction of the internal elastic membrane (asterisk).

the popliteal artery in addition to releasing of the aberrant muscle bundle. In the present case, we pathologically confirmed that the removed portion of the popliteal artery was severely occluded, accompanied by fibrosis and destruction of the internal elastic membrane without any findings of aneurysm or thrombus. Therefore, we considered that occlusion of the popliteal artery was brought by repetitive mechanical stimulation of aberrant muscle bundle. There is only one case in which not only the successful surgical reconstruction but the similar pathological finding also been reported on the paper. ${ }^{4)}$ In that case, the pathological finding showed acute occlusion of popliteal artery by thrombotic material. However, in the present case, the popliteal artery was occluded chronically with distal reconstruction via collateral artery judging from the clinical symptom and the finding of the CTA and pathological findings. We should deliberate whether we reconstruct the popliteal artery in addition to release of the aberrant muscle bundle due to the preoperative examination to circumvent the reoperation.

\section{ACKNOWLEDGEMENT}

The authors are indebted to Roderick J. Turner and 
Prof. J. Patrick Barron of the International Medical Communications of Tokyo Medical University for their review of this article.

\section{REFERENCES}

1) Stuart TP. Note on a Variation in the Course of the Popliteal Artery. J Anat Physiol. 1879; 13: 162.

2) Rich NM, Collins GJ Jr, McDonal PT, Kolzloff L,
Clagett GP, Collins JT. Popliteal vascular entrapment: its increasing interest. Arch Surg. 1979; 114: 1377-84.

3) Rochier AL, Sumpio BE. Variant of popliteal entrapment syndrome involving the lateral head of the gastrocnemius muscle: a case report. Ann Vasc Surg. 2009; 23: 535.e5-e9.

4) Schurmann G, Mattfeldt T, Hofmann W, Hohenberger P, Allenberg JR. The popliteal entrapment syndrome: presentation, morphology and surgical treatment of 13 cases. Eur J Vasc Surg. 1990; 4: 223-31. 